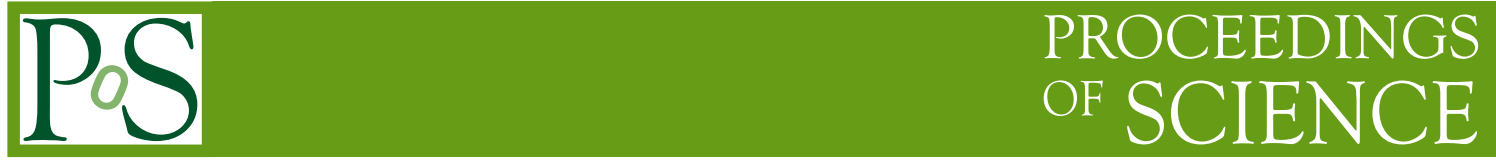

\title{
Hadronization in SU(N) Gauge Field Theory
}

\section{Andrey V. Koshelkin*}

Moscow Institute for Physics and Engineering, Kashirskoye sh., 31, 115409 Moscow, Russia E-mail: A Kosh@internets.ru

The bosonization of the strong interacting matter as a process of arising observable hadrons is studied in terms of the phase transition of the second kind. The spectrum of bosons which is free from the zero point energy is derived. The calculated boson mass is found to depend selfconsistently on both the amplitude of a gauge field and quark mass. In the framework of the quasi-classical model[10, 11] a hadron mass is calculated in the case of bosonization into pions.

Sixth International Conference on Quarks and Nuclear Physics,

April 16-20, 2012

Ecole Polytechnique, Palaiseau, Paris

\footnotetext{
* Speaker.
} 


\section{Introduction}

Since observable particles are colorless the process of the hadronization plays a key role in QCD. The problem complicates when hadrons are mesons, since such particles are governed by the Bose-Einstein statistics while they consist of interacting fermions.

The bosonization generally means a description of fermionic systems in terms of the collective boson degrees of freedom[1, 2]. It is preferable to bosonate a fermionic system by solving the motion equation for interacting fermions. Although this way is the most correct and elegant it has been still made in the case of the Minkowski space-time $(1+1)$. Following such technique the bosonization of the strong interaction matter is considered many times [3, 4, 5, 6, 7, 8, 9]. The heart of the method developed in the papers [3, 4, 5, 6, 7, 8, 9] is existence of the so-called flux tube, when the strong interacting matter is suggested to be in the condition of the longitudinal dominance and transverse confinement in the Minkowski space-time.

In the present paper the bosonization as a process of arising observable hadrons is studied in terms of the QCD lagrangian in the standard $(1+3)$ Minkowski space-time without any prior fragmentation. Considering the bosonization as the equilibrium phase transition of the second kind, the boson spectrum is derived in the quasi-classical approximation far from the fluctuation region of the transition. The obtained spectrum is free from the zero point energy. When the confinement phase is governed by the equations of the self-consistent quasi-classical model[10, 11] a boson mass is calculated provided that a quark-gluon plasma is bosonated into the lightest bosons (pions).

\section{General equations}

The gauge invariant action $\mathscr{A}$ in the $\mathrm{SU}(\mathrm{N})$ field theory is[12]:

$$
\mathscr{A}=\int d^{4} x\left\{\left[\bar{\psi}(x) \gamma^{k}\left(i \partial_{k}+g T_{a} A_{k}^{a}\right) \psi(x)-\bar{\psi}(x) m \psi(x)\right]-\frac{1}{16 \pi} F_{\mu \nu}^{a} F_{a}^{\mu v}\right\},
$$

where $F_{\mu \nu}^{a}$ is the tensor of the non-abelian gauge filed which is given by the expression:

$$
F_{\mu \nu}^{a}=\partial_{\mu} A_{v}^{a}-\partial_{v} A_{\mu}^{a}+g f_{b c}^{a} A_{\mu}^{b} A_{v}^{c}
$$

The action integral (2.1) generates the energy-momentum tensor

$$
\begin{aligned}
& T^{\mu v}=\frac{i}{2}\left\{\bar{\Psi}(x) \gamma^{\mu} \partial^{v} \Psi(x)-\bar{\Psi}(x) \gamma^{\mu} \overleftarrow{\partial}^{v} \Psi(x)\right\}+g\left(J^{a}(x)\right)^{\mu} A_{a}^{v}(x) \\
& +\frac{1}{4 \pi}\left\{-F_{a}^{\mu i}(x)\left(F^{a}\right)_{i}^{v}(x)+\frac{\mathscr{G}^{\mu v}}{4} F_{a}^{i k}(x) F_{i k}^{a}(x)\right\}
\end{aligned}
$$

and the motion equations:

$$
\partial_{\mu} F_{a}^{v \mu}(x)-g \cdot f_{a b}{ }^{c} A_{\mu}^{b}(x) F_{c}^{v \mu}(x)=-g J_{a}{ }^{v}(x), \quad J_{a}{ }^{v}(x)=\bar{\Psi}(x) \gamma^{v} T_{a} \Psi(x),
$$


In this way, the fermion fields $\Psi(x), \bar{\Psi}(x)$ are governed by the Dirac equation:

$$
\left\{i \gamma^{\mu}\left(\partial_{\mu}+i g \cdot A_{\mu}^{a}(x) T_{a}\right)-m\right\} \Psi(x)=0 .
$$

In Eqs.(2.1) - (2.5) we introduce the following notations; $m$ is a fermion mass, $g$ is the coupling constant; $\gamma^{\nu}$ are the Dirac matrices, $x \equiv x^{\mu}=\left(x^{0} ; \mathbf{x}\right)$ is a vector in the Minkowski space-time; $\partial_{\mu}=(\partial / \partial t ; \nabla)$; the Roman letters numerate a basis in the space of the associated representation of the $S U(N)$ group, so that $a, b, c=1 \ldots N^{2}-1$. We use the signature $\operatorname{diag}\left(\mathscr{G}^{\mu v}\right)=(1 ;-1 ;-1 ;-1)$ for the metric tensor $\mathscr{G}^{\mu \nu}$. The line over $\Psi$ means the Dirac conjugation. Summing over any pair of the repeated indices is implied. The symbols $T_{a}$ in Eqs.(2.1) - (2.5) are the generators of the $S U(N)$ group, while $f_{a b}{ }^{c}$ are the structure constants therein; $\delta_{a b}$ is the Kroneker symbol.

We assume that the field $A_{v}^{a}(x)$ depends on coordinates via some scalar function $\varphi(x)$ in the Minkowski space-time which is normally named as the eikonal:

$$
A_{a}^{v}(x)=A_{a}^{v}(\varphi(x)) .
$$

Let the axial gauge be for the field $A_{\mu}^{a}(x)$ :

$$
\partial^{\mu} A_{\mu}^{a}=0 ; \quad k^{\mu} \dot{A}_{\mu}^{a}=0
$$

where the dot over the letter means differentiation with respect to the introduced variable $\varphi$ while the vector $k^{\mu}=\partial^{\mu} \varphi(x)$.

The introduced vector $k^{\mu}$ indicates the direction along the eikonal, while Eq.(2.7) means the local transversion of the gauge field $A_{\mu}^{a}$.

Due to Eq.(2.6) the hamiltonian generated by the action (2.1) is explicitly independent of the time variably. This means that some stationary states of fermions, which energy is $\varepsilon(\vec{p})$, exist.

Then, the hamiltonian of interacting fermions can be written as follows:

$$
\mathscr{H}=\int d^{3} \vec{x} T^{00}=\sum_{\vec{p} ; \sigma, \alpha} \varepsilon(\vec{p})\left[n_{\sigma, \alpha}(\vec{p})+\left(1-\bar{n}_{\sigma, \alpha}(\vec{p})\right)\right]+\int d^{3} \vec{x} T_{g}^{00},
$$

where $T_{g}^{00}$ is the zeroth component of the momentum-energy tensor of the gauge field; $\left.n_{\sigma, \alpha}(\vec{p})\right)$ and $\left.\bar{n}_{\sigma, \alpha}(\vec{p})\right)$ are the occupation numbers of fermions and anti-fermions, respectively.

\section{Bosonization}

In studying the bosonization we assume the bosonization starts when the fermion vacuum is full such that the occupation number of both particles $n_{\sigma, \alpha}(\vec{p})$ and anti-particles $\bar{n}_{\sigma, \alpha}(\vec{p})$ are equal to unit:

$$
n_{\sigma, \alpha}(\vec{p})=1 ; \quad \bar{n}_{\sigma, \alpha}(\vec{p})=1 .
$$


As for the gluon field, we assume that the number of gluons is large $n_{g} \gg 1$ due to the selfinteraction of them. Since $n_{g} \gg 1$, the gluon field can be considered quasi-classically. Besides that fermion and gauge fields are suggested to be in equilibrium.

On the other hand, $A_{\mu}^{a}$ is self-interacting field that leads to the generation of new quanta of $A_{\mu}^{a}$ in spite of the equilibrium state. Then, creation of additional quanta on the background of the fullness of the fermion vacua has to result in arising new particles since the entropy is in maximum.

Let us consider the matter consisting of interacting quarks and gluons. We assume that the matter transits to the deconfinement phase so that bosons only arise as observable particles.

We present the gauge field as sum of two orthogonal terms in the group space such that $A_{\mu}^{a}$ has the following form:

$$
A_{\mu}^{a}(\varphi)=\mathscr{A}_{\mu}^{a}+e_{\mu}^{a} \Phi(\varphi) ; \quad \mathscr{A}_{\mu}^{a} e_{b}^{\mu}=0 ; \quad e_{\mu}^{a} e_{b}^{\mu}=-\delta_{b}^{a},
$$

where $\mathscr{A}_{\mu}^{a}$ is amplitude of the gauge field just before the phase transition. The amplitude $\mathscr{A}_{\mu}^{a}$ is taken to be constant in the Minkowski space-time, while $\Phi(\varphi)$ is a scalar function therein. The field $\Phi(\varphi)$ only is not to equal to zero in the deconfinement phase, and plays a role of the order parameter. We note that the presentation of $\mathscr{A}_{\mu}^{a}$ in the form given by Eq.(3.2) means that the phase transition is considered far from the fluctuation region.

Then, the gluon part of the momentum-energy tensor $T_{g}^{00}$, which is given by Eq.(2.8), is of the form:

$$
\begin{aligned}
& T_{g}^{00}=\frac{1}{16 \pi}\left\{4\left(N^{2}-1\right)\left(\partial^{0} \Phi\right)^{2}-2\left(N^{2}-1\right)\left(\partial^{v} \Phi\right)\left(\partial_{v} \Phi\right)+2 N g^{2} A^{2} \Phi^{2}\right. \\
& \left.+g^{2} f_{a}^{b c} f_{b_{1} c_{1}}^{a} \mathscr{A}_{b}^{v} \mathscr{A}_{c}^{\mu} \mathscr{A}_{v}^{b_{1}} \mathscr{A}_{\mu}^{c_{1}}+g^{2} N\left(N^{2}-1\right) \Phi^{4}\right\} ; \quad-A^{2} \equiv \mathscr{A}_{\mu}^{a} \mathscr{A}_{a}^{\mu}
\end{aligned}
$$

We study the situation when the density $n_{0}$ of particles governed by the field $\Phi$ is not too large, so that $n_{0}^{1 / 3} \lambda_{C} \ll 1$, where $\lambda_{C}=1 / M$ is the Compton wave length of the particle with a mass $M$.

Then, the last term in Eq.(3.3) is small. As a result, taking into account the inequality $n_{0}^{1 / 3} \lambda_{C} \ll$ 1 , we rewrite the hamiltonian given by Eq.(2.8) by the following way:

$$
\begin{aligned}
& \mathscr{H}=\sum_{\vec{p} ; \sigma, \alpha} \varepsilon(\vec{p})+\frac{1}{16 \pi} \int d^{3} \vec{x}\left\{4\left(N^{2}-1\right)\left(\partial^{0} \Phi\right)^{2}-2\left(N^{2}-1\right)\left(\partial^{v} \Phi\right)\left(\partial_{v} \Phi\right)+2 N g^{2} A^{2} \Phi^{2}+\right. \\
& \left.g^{2} f_{a}^{b c} f_{b_{1} c_{1}}^{a} \mathscr{A}_{b}^{v} \mathscr{A}_{c}^{\mu} \mathscr{A}_{v}^{b_{1}} \mathscr{A}_{\mu}^{c_{1}}\right\} .
\end{aligned}
$$

By changing $\Phi \rightarrow \vec{\Phi}$, the relations (3.4) is easy generalized to the case when the field $\vec{\Phi}$ is the triplet of pseudoscalar mesons, where $\vec{\Phi}$ is the vector in the isospace.

We expand $\vec{\Phi}(\varphi)$ over the whole set of the plane waves: 


$$
\begin{aligned}
& \vec{\Phi}(\varphi)=\sum_{\vec{q}} \sqrt{\frac{8 \pi}{V\left(N^{2}-1\right) \omega(\vec{q})}}\left\{\vec{e} c(\vec{q}) \exp (-i q x)+\vec{e}^{*} c^{\dagger}(\vec{q}) \exp (i q x)\right\}, \\
& \omega(\vec{q})=\sqrt{\vec{q}^{2}+M^{2}}, M^{2}=\frac{N g^{2} A^{2}}{\left(N^{2}-1\right)} ; \vec{e} \vec{e}^{*}=1,
\end{aligned}
$$

where $\vec{e}$ is the unit vector in the isospace; $c(\vec{q})$ and $c^{\dagger}(\vec{q})$ are the operators of annihilation and creation of the on-shell particle $\left(q^{2}=M^{2}\right)$ with the 4-momentum $q=(\omega(\vec{q}) ; \vec{q})$. The operators $c(\vec{q})$ and $c^{\dagger}(\vec{q})$ satisfy the standard Bose-Einstein commutative relations.

Let us substitute the expansion given by Eq.(3.5) into the formula (3.4) and average the obtained relation over the vacuum of the field $\vec{\Phi}$. As a result, we derive the energy of the particles governed by the pseudoscalar field $\vec{\Phi}$ :

$$
E=\sum_{\vec{q}} \omega(\vec{q})<c^{\dagger}(\vec{q}) c(\vec{q})>+\left\{\frac{1}{2} \sum_{\vec{q}} \omega(\vec{q})+\sum_{\vec{p} ; \sigma, \alpha} \varepsilon(\vec{p})+g^{2} f_{a}^{b c} f_{b_{1} c_{1}}^{a} \mathscr{A}_{b}^{v} \mathscr{A}_{c}^{\mu} \mathscr{A}_{v}^{b_{1}} \mathscr{A}_{\mu}^{c_{1}}\right\}
$$

where the angle brackets mean averaging over the pseudoscalar vacuum.

Since the vacuum of arising pseudoscalar particles should be empty the term in the curl brackets has to be equal to zero. This condition (the zero magnitude of the curl bracket in Eq.(3.6)) determines the spectrum $\varepsilon(\vec{p})$ of quarks via the gauge field $\mathscr{A}_{\mu}^{c}$ just before the phase transition. We should note here that the last term in the curl bracket should be negative.

As a result, we obtain the energy spectrum of scalar hadrons:

$$
E_{h}=\sum_{\vec{q}} \omega(\vec{q}) N_{h}(\vec{q}) ; \quad \omega(\vec{q})=\sqrt{\vec{q}^{2}+M^{2}}
$$

provided that

$$
\left\{\frac{1}{2} \sum_{\vec{q}} \omega(\vec{q})+\sum_{\vec{p} ; \sigma, \alpha} \varepsilon(\vec{p})+g^{2} f_{a}^{b c} f_{b_{1} c_{1}}^{a} \mathscr{A}_{b}^{v} \mathscr{A}_{c}^{\mu} \mathscr{A}_{v}^{b_{1}} \mathscr{A}_{\mu}^{c_{1}}\right\}=0
$$

where $N_{h}(\vec{q})$ is the number of the on-shell hadrons with the 4-momentum $q=(\omega(\vec{q}) ; \vec{q})$.

\section{Bosonization in quasi-classical model}

Let us apply the results obtained in the previous sections to calculation of a boson mass in terms of the self-consistent quasi-classical model developed in Ref.[10, 11]. In this case the convolution in Eqs.(3.3), (3.4) is equal to[11]:

$$
-f_{a}^{b c} f_{b_{1} c_{1}}^{a} \mathscr{A}_{b}^{v} \mathscr{A}_{c}^{\mu} \mathscr{A}_{v}^{b_{1}} \mathscr{A}_{\mu}^{c_{1}}=\left(N^{2}-1\right) \sum_{\sigma \alpha} \int \frac{d^{3} p}{p^{(0)}(2 \pi)^{3}} .
$$


Then, the boson mass $M$ is given by a formula:

$$
M^{2} \approx \frac{2 N N_{f} \alpha_{s}}{2|C| \pi} Q^{2} ; \quad \alpha_{s}=\frac{g^{2}}{4 \pi}, \quad C=-f_{a}^{b c} f_{b_{1} c_{1}}^{a} \cos \left(\varphi_{b}-\varphi_{b_{1}}\right) \cos \left(\varphi_{c}-\varphi_{c_{1}}\right)>0,
$$

where $\alpha_{s}$ is the strong interaction coupling constant, $Q$ is the transferred momentum corresponding to the confinement-deconfinement phase transition which is of the order of the phase transition temperature. The parameters $\varphi_{b, c, b_{1}, c_{1}}$ are the phases of the amplitudes $\mathscr{A}_{b, c, b_{1}, c_{1}}^{v}$ which are fixed such that the convolution $C$ is negative.

In the case $N_{f}=2 ; N=3$, we have

$$
M \approx \sqrt{\frac{12 \alpha_{s}}{\pi}} Q
$$

The last formula establishes relation of the hadron mass $M$ to the momentum of interacting particles in the matter which depends strongly on the matter temperature $T$.

Setting $T=Q=213 \mathrm{MeV}[13]$, we have $\alpha_{s}=0.12$. As a result we obtain:

$$
M \approx 144 \mathrm{MeV} ;|C| \sim 1
$$

that corresponds to the pion mass.

Although the derived pion mass is very nearly to the tabulated data Eq.(4.3) should be mainly treated as the formula giving the relation of a hadron mass to the temperature of phase transition. In particular, when the phase transition temperature is around $200 \mathrm{MeV}$ the result for the mass of observable particles is found to be correct.

\section{References}

[1] M.Stone. Bosonization, World Scientific, Singapore, 1994.

[2] E.H.Fradkin, F.A.Schaposnik, Phys. Lett., B338 253 (1994).

[3] A.Casher, J.Kogut, and L.Susskind, Phys. Rev. D10, 732 (1974).

[4] S.Coleman, Ann. Phys. 101, 239 (1976). C.Y.Wong, Phys.Rev. C 80054917 (2009).

[5] S.Coleman, R.Jackiw, L.Susskind, Ann. Phys. 98, 267 (1975).

[6] J.D.Boerken, Phys. Rev. D 27, 140 (1983)

[7] C.Y.Wong, Phys. Rev. C80, 034908 (2009).

[8] C.Y.Wong, Phys. Rev. C80, 054917 (2009).

[9] C.Y.Wong, arXiv:1001.1691v3[hep-ph].

[10] A.V.Koshelkin, Phys. Lett. B 683 (2010) 205.

[11] A.V.Koshelkin, Phys. Lett. B 696 (2011) 539.

[12] M.E.Peskin, D.V.Schroeder, An introduction to Quantum Field Theory. Addison-Wesley Publishing Company, 1995.

[13] D.E.Kharzeev, Nucl.Phys. A 699 (2002) 95; D.E.Kharzeev, J.Raufeisen, nucl-th/0206073, v1, 28 June, 2002. 